\title{
Evaluation Method for Multi-Operator and Multi-Robot Teleoperation Systems
}

\author{
Guanhua Feng ${ }^{\dagger}$, Wenhao Li ${ }^{\dagger \dagger *}$ and Heng Zhang ${ }^{\dagger \ddagger}$ \\ ${ }^{\dagger}$ Institute of Mechanics, Chinese Academy of Sciences \\ No.15 Beisihuanxi Road, Beijing, P.R.China 100190 \\ ${ }^{\ddagger}$ School of Engineering Sciences, University of Chinese Academy of Sciences \\ No.19(A) Yuquan Road, Shijingshan District, Beijing, P.R.China 100049 \\ *Email: liwenhao@imech.ac.cn (Corresponding author)
}

\begin{abstract}
This paper focuses on the topic of evaluation method for Multi-Operator and Multi-Robot (MOMR) teleoperation systems. First, a new evaluation method for MOMR teleoperation systems was proposed, and the corresponding grouping method of terminals and robots in system and its group evaluation method we proposed was discussed. Second, ten kind of universal capabilities covering 128 functional items and 64 quantitative items were summarized for four operation mode evaluation in the process of group evaluation. Then group evaluation capabilities and items covering 11 functional items and 10 quantitative items were summed up for the comprehensive evaluation of Single-Operator Multi-Robot (SOMR) operation mode group (SOMROMG), Multi-Operator Single-Robot (MOSR) operation mode group (MOSROMG), and Multi-Operator Multi-Robot (MOMR) operation mode group (MOMROMG). Then evaluation capabilities and items covering 19 functional items and 10 quantitative items were also listed for the evaluation of intermediate service node of MOMR operation mode. Finally, through example the effectiveness of the proposed method was validated.

Keywords-evaluation method; Multi-Operator and Multi-Robot (MOMR) teleoperation systems; universal capabilities; grouping method; evaluation items
\end{abstract}

\section{INTRODUCTION}

Teleoperation, also known as remote control of robots, is a hot topic in the field of information technology now and a complex multidisciplinary application technology involving robotics, computer science, instruments, dynamics and control, etc [1]. The operating platform based on it can not only complete the information integration tasks such as data processing and image display, providing decision-making help for the operator to participate in the task, also effectively apply various operating modes to the task flow to realize the task safely and quickly [2], [3]. Therefore, teleoperation system of robots is widely applied in deep sea exploration, space exploration and harsh environment operation, etc [3], [4].

In practical applications, a teleoperation task may require multiple operators to cooperatively and remotely control multiple robots to perform cooperation, and operators/robots may be located in different positions, that is a Multi-Operator and Multi-Robot (MOMR) teleoperation systems. In the whole task process, all operator terminals and robots are connected to a communication system, and there is a cooperate teleoperation technology problem between operator and operator, operation decision maker and decision maker. All operators need to complete cooperatively the teleoperation task under the command of operation decision maker [5]-[7]. The reliability of cooperative operation among operator terminals, the decisionmaking and interoperability capability among operators, and the decision-making capability of different regions are crucial factors affecting the teleoperation effectiveness, flexibility and task security of robots [8], [9].

However, for the completion of the teleoperation task, the execution result can only be seen after the entire task is completed; and for the task execution capability and interoperability of various terminals, the effect of operational environment in different region on the respond speed of terminal, etc. cannot be evaluated in a timely and effective manner during the execution of the entire task and after the completion of the task. So this paper introduces how to evaluate a MOMR teleoperation systems by its previous task(s) data and establishes a universal evaluation systems for MOMR teleoperation systems. In the paper, Section II treats evaluation method for MOMR teleoperation systems. Evaluation example of a teleoperation systems is analyzed based on the proposed method in Section III. The last Section IV gives the conclusion.

\section{Evaluation Method}

We firstly give the evaluation method folw chart directly for a MOMR teleoperation systems shown in Fig. 1. From the folw chart we know, specific teleoperation task(s) data of each group after terminals and robots grouping is evaluated and statistical results are used for the further MOMR teleoperation systems evaluation. Then steps of the evaluation method are discussed in detailed. In Section II-B, we introdue the grouping method for operator terminals and robots of a MOMR teleoperation systems based on specific teleoperation task flow. The next Section II-C and II-D discusses the evaluation method of each group and the MOMR teleoperation systems evaluation method.

\section{A. MOMR Teleoperation Systems}

MOMR teleoperation systems indicates that $M(M>1)$ operator terminals with different delay environments at the same/different location and homogeneous/heterogeneous $N(N>1)$ operation objects (such as robots, manipulator, etc.) 


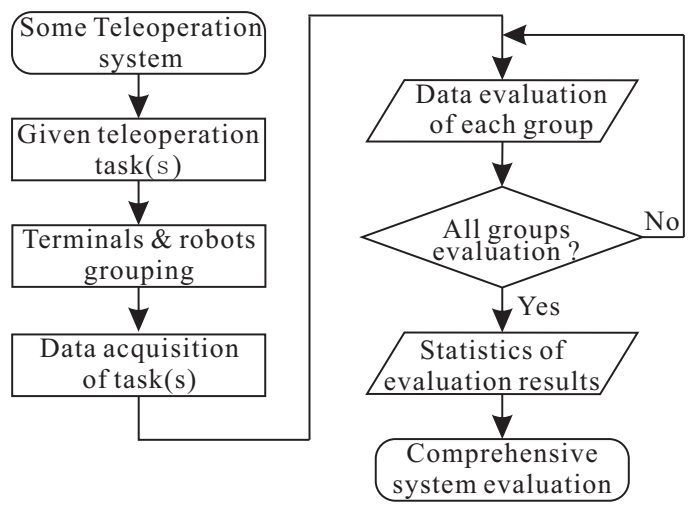

Fig. 1. Evaluation method flow chart for MOMR teleoperation systems

distributed at the same/different site are connected to the same manipulate network, and time synchronization is maintained continually among all terminals, and between terminals and objects; based on the above, $N$ objects are manipulated remotely by $M$ terminals to collaborate to complete a particular teleoperation task.

\section{B. Terminals \& Robots Grouping}

According to the flow of teleoperation task(s), $M(M>1)$ operator terminals and $N(N>1)$ operation objects (robots) of a MOMR teleoperation systems are grouped before the execution of the teleoperation task(s), each of which includes at least one terminal and one object. The aboved grouping specifically refers to that one/more terminals and one/more object are grouped into a task operation mode group we called according to their mapping relationship of remote control, each of which includes at least one terminal and one object. Where the operator terminal(s) grouped in a task operation mode group is called an operator terminal group, and the operation object(s) is called an operation object group; each operator terminal group has its corresponding operation object group and the total number of task operation mode groups is equal to the total number of operator terminal groups or operation object groups. And according to the number of terminal(s) and object(s) in each task operation mode group, task operation mode group can be divided into four types including Single-Operator Single-Robot (SOSR) operation mode group (SOSROMG), Single-Operator Multi-Robot (SOMR) operation mode group (SOMROMG), Multi-Operator SingleRobot (MOSR) operation mode group (MOSROMG) and Multi-Operator Multi-Robot (MOMR) operation mode group (MOMROMG).

SOSR operation mode is that one operator terminal and one operation object (such as robots, manipulator, etc.) are connected to the same manipulate network, and time synchronization is maintained continually between them to remotely complete a particular teleoperation task. Similarly, operation mode of SOMR with one terminal and $n_{1}\left(n_{1} \geq 1\right)$ objects, MOSR with $m_{1}\left(m_{1} \geq 1\right)$ terminals and one object, MOMR with $m_{1}\left(m_{1} \geq 1\right)$ terminals and $n_{1}\left(n_{1} \geq 1\right)$ objects can be known.
When grouping specifically, firstly, according to the flow of the loaded $i$-th $(k=1,2, \ldots, R, R$ is the total number of effective teleoperation task(s)) teleoperation task and combined with the actual situation of all terminals and objects, terminals and objects of MOMR teleoperation system are divided into groups of $v_{i}\left(v_{i} \geq 1\right)$, that is, all terminals are divided into operator terminal groups of $v_{i}\left(v_{i} \geq 1\right)$, and all objects are divided into operator object groups of $v_{i}\left(v_{i} \geq 1\right)$. Secondly, the $v_{i}$ groups of operator terminals and operation objects with the corresponding mapping relationship are further divided into SOSROMG of $w_{i}\left(w_{i} \geq 0\right)$, SOMROMG of $x_{i}\left(x_{i} \geq 0\right)$, MOSROMG of $y_{i}\left(y_{i} \geq 0\right)$, MOMROMG of $z_{i}\left(z_{i} \geq 0\right)$, and

$$
v_{i}=w_{i}+x_{i}+y_{i}+z_{i}
$$

Note that when dividing groups, all terminals mapping the same object in the $M$ operator terminals are divided into one operator terminal group, and all objects remotely manipulated by the same terminal in the $N$ operation objects is divided into one operation object group, that is, one terminal may be divided into multiple operator terminal groups, and any object can only be grouped into one operation object group.

\section{Evaluation Method of Group}

MOMR teleoperation systems is mainly developed for multiple operators to control multiple space robots to fulfill various space tasks. To obtain a better systems evaluation results, it's firstly necessary to develop the method of each task operation mode group.

1) Data Acquisition of Task(s): During the entire process of teleoperation task(s) execution, when remotely manipulating one/more objects in the $n_{2}$ operation objects, one/more terminals in the $m_{2}$ operator terminals generate the teleoperation request and send it to the intermediate service node, and then generate the corresponding teleoperation instructions and execution time for objects to complete the operation request after receiving the permission from the intermediate service node. Similarly, one/more terminals in the $M$ operator terminals of a MOMR teleoperation systems remotely manipulate one/more objects in the $N$ operation objects only after the permission from the intermediate service node. Based on the above, complete task(s) data of each task operation mode group can be acquired. A large number of teleoperation tasks should be executed for the achievement of better evaluation of each group.

2) Evaluation Method of Group: Based on the combination of the acquired groups data and all quantitative and functional evaluation items shown in the following section, the group evaluation result indicated by $E_{i}$ of $i$-th $(k=1,2, \ldots, R)$ is given by Eq. (1). Where assume that the $j$-th universal capability has $m$ functional evaluation items and $n$ quantitative evaluation items.

$$
E_{i}=\sum_{j=1}^{S}\left(\boldsymbol{\alpha}_{j}^{\mathrm{T}} \mathbf{P}_{j}+\boldsymbol{\beta}_{j}^{\mathrm{T}} \mathbf{Q}_{j}\right)
$$

Where $\boldsymbol{\alpha}_{j}$ and $\boldsymbol{\beta}_{j}$ are weight matrix of functional and quantitative evaluation items respectively, and the value of their 
element $\alpha_{j k}(k=1,2, \ldots, f)$ and $\beta_{j l}(l=1,2, \ldots, g)$ is determined by the relative importance among all evaluation items.

$$
\begin{aligned}
\boldsymbol{\alpha}_{j} & =\left[\alpha_{j 1}, \alpha_{j 2}, \ldots, \alpha_{j f}\right]^{\mathrm{T}} \\
\boldsymbol{\beta}_{j} & =\left[\beta_{j 1}, \beta_{j 2}, \ldots, \beta_{j g}\right]^{\mathrm{T}}
\end{aligned}
$$

and

$$
\begin{gathered}
0 \leq \alpha_{j k}, \beta_{j l} \leq 1 ; \\
\sum_{k=1}^{f} \alpha_{j k}+\sum_{l=1}^{g} \beta_{j l}=1 .
\end{gathered}
$$

$\mathbf{P}_{j}$ and $\mathbf{Q}_{j}$ are evaluation value of functional and quantitative evaluation items respectively, and the value of their element $p_{j k}(k=1,2, \ldots, f)$ and $p_{j l}(l=1,2, \ldots, g)$ is equal to 0 or 1 , determined by whether the criteria is satisfied.

$$
\begin{aligned}
& \mathbf{P}_{j}=\left[p_{j 1}, p_{j 2}, \ldots, p_{j f}\right]^{\mathrm{T}} ; \\
& \mathbf{Q}_{j}=\left[q_{j 1}, q_{j 2}, \ldots, q_{j g}\right]^{\mathrm{T}},
\end{aligned}
$$

and

$$
\begin{gathered}
p_{j k}= \begin{cases}1, & \text { if } k \text {-th item is satisfied } \\
0, & \text { otherwise. }\end{cases} \\
q_{j l}= \begin{cases}1, & \text { if } l \text {-th item is in target range } \\
0, & \text { otherwise. }\end{cases}
\end{gathered}
$$

Evaluation of SOSR operation mode is completed mainly from 8 universal capabilities shown in Fig. 2, and each capability has its corresponding $m$ functional evaluation items and $n$ quantitative evaluation items shown in Fig. 3 . Where items marked with "*" are the quantitative evaluation items whose quantized value directly reflects the evaluation results of the corresponding evaluation item, and unmarked items are the functional evaluation items whose evaluation results are reflected by whether to has the function of the corresponding item. SOSROMG is evaluated according to Eq. (1), and iteration evaluation result is indicated by $E_{i}^{1}$.

Similarly, SOMR, MOSR, and MOMR operation mode can also be evaluated according to Eq. (1), and some universal capabilities or evaluation items for the evaluation of SOMROMG, MOSROMG, and MOMROMG are added on the basis of that of SOSROMG, shown in Fig. 2,; Besides, group evaluation capabilities and items are given for the comprehensive evaluation of SOMROMG, MOSROMG, and MOMROMG, and evaluation capabilities and items for intermediate service node of MOMR operation mode are also added, shown in Fig. 4. Their result are indicated by $E_{i}^{2}, E_{i}^{3}$, and $E_{i}^{4}$ respectively.

\section{Evaluation Method for MOMR Teleoperation Systems}

1) Statistics of Evaluation Results: Before we give the comprehensive MOMR teleoperation evaluation, we first must complete evaluation of all $R$ tasks data acquired in Section II-C1 based on evaluation method given in Section II-C2. Then Statistics of the above evaluation results of all groups can be further obtained.

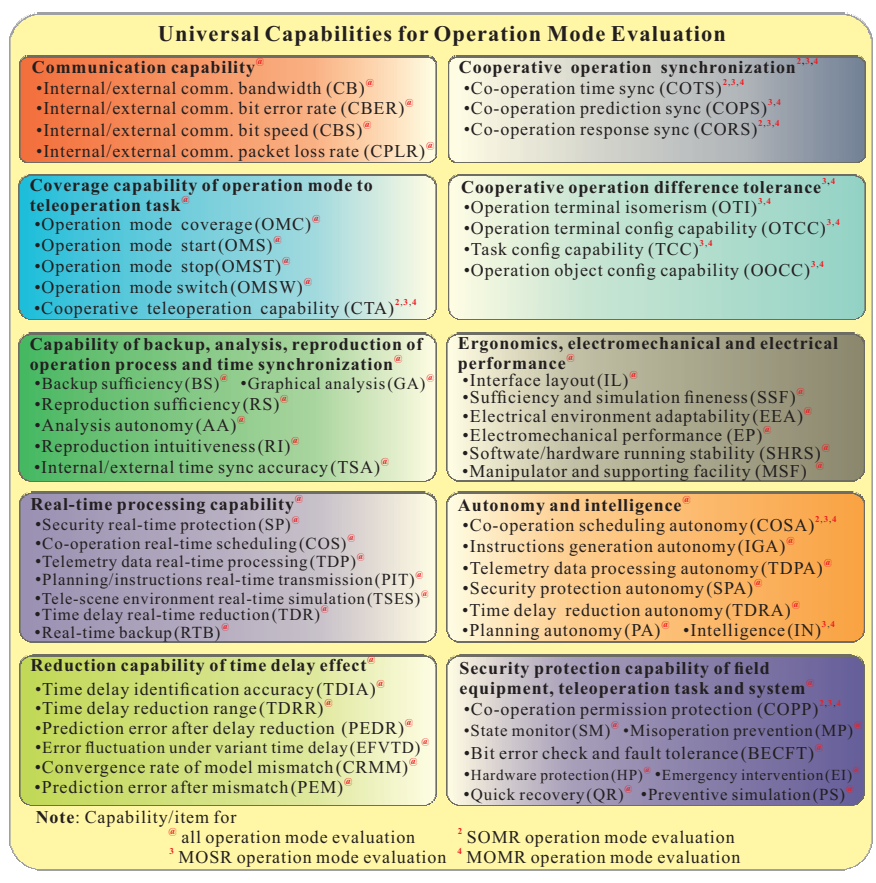

Fig. 2. Universal capabilities for opertion mode evaluation

2) Comprehensive Systems Evaluation: The comprehensive MOMR teleoperation systems evaluation can be defined as follows according the statistics results of $R$ tasks data, and its evaluation result is indicated by $E_{R}$.

$$
E_{R}=\sum_{i=1}^{R} c_{i} \mathbf{G}_{i} \mathbf{E}_{i}
$$

Where $c_{i}\left(0 \leq c_{i} \leq 1\right)$ is the complexity coefficient of $i$ th teleoperation task and is used to reflect the complexity of a teleoperation task. $\mathbf{G}_{i}$ and $\mathbf{E}_{i}$ are the grouping coefficient matrix and evaluation result matrix of groups of the $i$-th teleoperation taskrespectively, and

$$
\begin{gathered}
\mathbf{G}_{i}=\left[\frac{w_{i}}{v_{i}}, \frac{x_{i}}{v_{i}}, \frac{y_{i}}{v_{i}}, \frac{z_{i}}{v_{i}}\right] ; \\
\mathbf{E}_{i}=\left[E_{i}^{1}, E_{i}^{2}, E_{i}^{3}, E_{i}^{4}\right]^{\mathrm{T}} .
\end{gathered}
$$

\section{EXAMPLE}

In order to validate the aboved method, the comprehensive evaluation of a Multi-Operator and Single-Robot (MOSR) teleoperation systems shown in Fig. 5 is carried out. The systems is consist of six units including data control and management unit, behaviors simulation unit of objective response, terminal 1, terminal 2, terminal 3 and image processing unit. Based on its teleoperation tasks data acquired, the systems evaluation is completed according to universal capabilities for MOSR operation mode and capabilities and items for MOSROMG shown in Fig. 2 - 4. Given space limitations, this paper only presents two of universal capabilities for systems evaluation as follows, including communication capability, and coverage capability of operation mode to teleoperation task. 


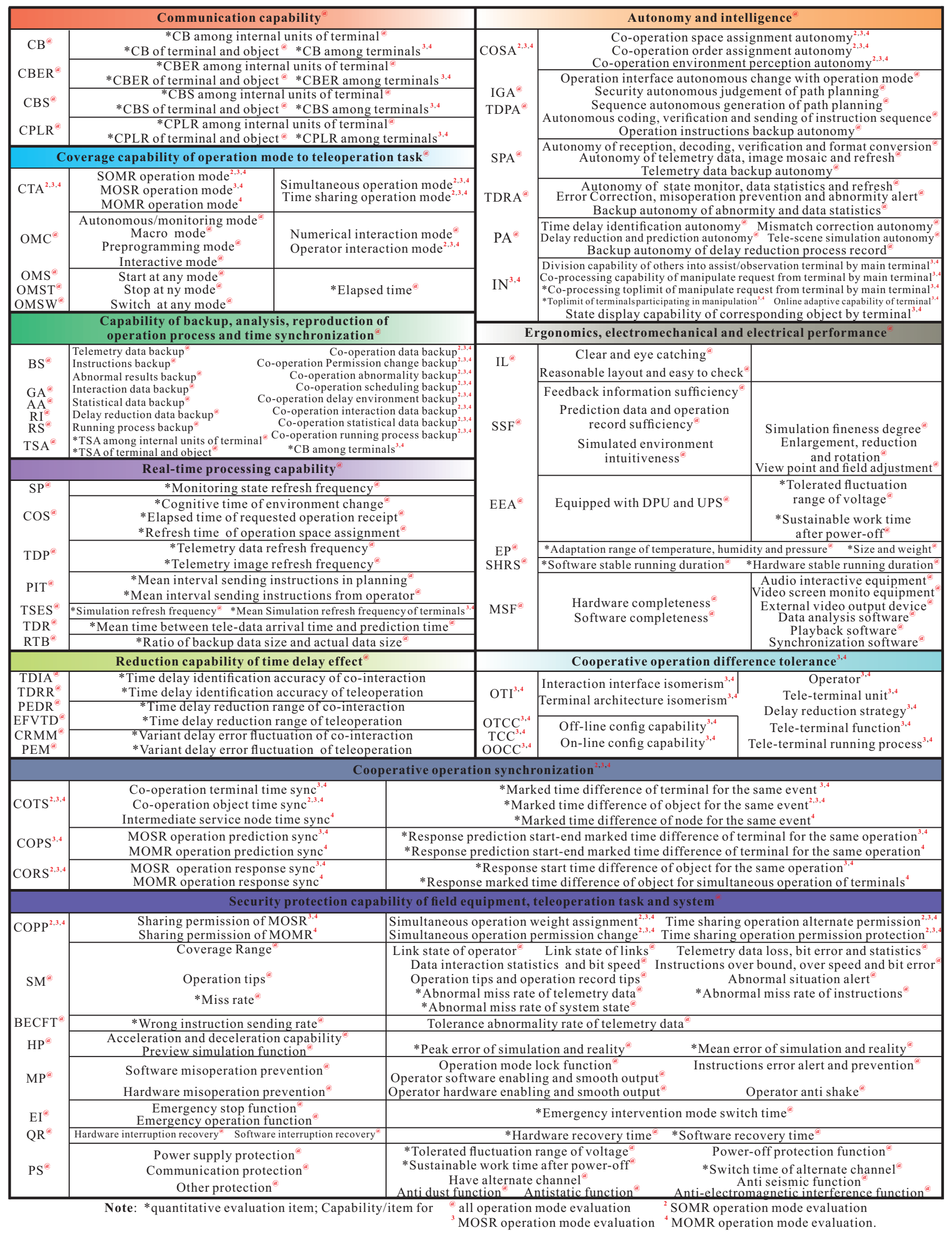

Fig. 3. Functional/quantitative evaluation items for universal capabilities 


\begin{tabular}{|c|c|c|}
\hline \multicolumn{3}{|r|}{ Group Evaluation Capability \& Item It, $^{2,3}$} \\
\hline Capability & Evaluation Content & Evaluation Item \\
\hline \multirow{3}{*}{$\begin{array}{l}\text { Extensibility } \\
\text { of Group }\end{array}$} & Extensibility for terminal $1^{3,4}$ & 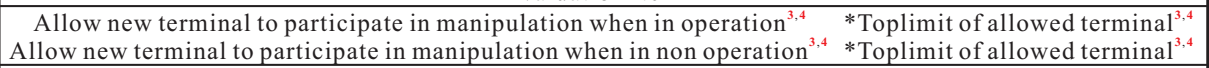 \\
\hline & Extensibility for object ${ }^{2,4}$ & 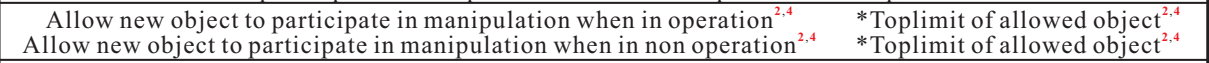 \\
\hline & Extensibility for software ${ }^{2,3,4}$ & Extensibility for software module of other functions $\mathrm{s}^{2,3,4}$ \\
\hline \multirow{4}{*}{$\begin{array}{l}\text { Difference Tolerance } \\
\quad \text { of Group }\end{array}$} & Tolerance for terminal $l^{3,4}$ & $\begin{array}{cc}\text { Tolerance for multiple heterogeneous terminals } & \text { *Toplimit of heterogeneous terminal } 1^{3,4} \\
\text { Allow any terminal to main terminal } & \text { *Toplimit of assist/obsetvation terminal }\end{array}$ \\
\hline & Tolerance for object ${ }^{2,4}$ & Tolerance for multiple different function/config objects ${ }^{2,4} *$ Toplimit of different function/config objects ${ }^{2,4}$ \\
\hline & Tolerance for delay ${ }^{2,3,4}$ & 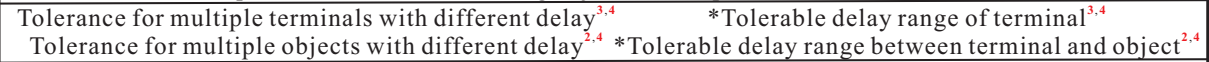 \\
\hline & Tolerance for task $\mathrm{tan}^{2,3,4}$ & Tolerance for tasks with different complexity ${ }^{2,4} \quad *$ Tolerable complexity range of task ${ }^{2,4}$ \\
\hline \multicolumn{3}{|c|}{ Evaluation Capability \& Item for Intermediate Service Node of MOMR Operation Mode } \\
\hline Capability & Evaluation Content & \begin{tabular}{|l} 
Evaluation Item \\
\end{tabular} \\
\hline \multirow{4}{*}{$\begin{array}{l}\text { Autonomy and } \\
\text { intelligence }\end{array}$} & $\begin{array}{l}\text { Multi objets/tasks } \\
\text { support capability }\end{array}$ & $\begin{array}{ll}\text { Grouping capability for terminals \& objects } & \text { *Toplimit of terminals when grouping } \\
& \text { *Toplimit of objects wshen grouping }\end{array}$ \\
\hline & $\begin{array}{l}\text { Autonomy of state judgment, } \\
\text { reception and sending }\end{array}$ & $\begin{array}{l}\text { Judgment autonomy of operating state of terminal/object } \\
\begin{array}{ll}\text { Reception autonomy of operating state from terminal/object } \\
\text { Sending autonomy of operating state of all terminals/objects to authorized terminal }\end{array} \\
\begin{array}{ll}\text { Storage/replacement autonomy of operating state from terminal/object } & \text { Sending autonomy of state infor. to all terminals }\end{array}\end{array}$ \\
\hline & $\begin{array}{c}\text { Response and sending autonomy } \\
\text { of manipulate request }\end{array}$ & $\begin{array}{ll}\text { Autonomy of request response from terminal } & \text { Sending autonomy of request permission to terminal } \\
\end{array}$ \\
\hline & $\begin{array}{c}\text { Autonomy of transmission delay } \\
\text { monitoring and maintenance }\end{array}$ & Monitoring autonomy of transmission delay $\quad$ Maintenance autonomy of transmission delay \\
\hline \multirow{3}{*}{$\begin{array}{l}\text { Real-time processing } \\
\text { capability }\end{array}$} & $\begin{array}{c}\text { Real-time of state judgment, } \\
\text { reception and sending }\end{array}$ & $\begin{array}{l}\text { *State judgment refresh frequency of terminal/object } * \text { Mean time difference between state sending and receiving } \\
\text { * Mean elapsed time of state reception from terminal/object } \\
\text { * Mean elapsed time of state sent to all terminals/objects }\end{array}$ \\
\hline & \begin{tabular}{c|}
$\begin{array}{c}\text { Real-time response and sending } \\
\text { of manipulate request }\end{array}$ \\
\end{tabular} & *Mean elapsed time of request response from terminal $\quad$ *Mean elapsed time of sending request permission to terminal \\
\hline & $\begin{array}{c}\text { Real-time maintenance of } \\
\text { transmission delay }\end{array}$ & *Maintenance refresh frequency of delay stored in intermediate service node \\
\hline \multirow[b]{2}{*}{$\begin{array}{l}\text { Synchronization } \\
\text { capability }\end{array}$} & Time sync capability & *Time difference of sending-reception time difference and measured delay \\
\hline & Response sync capability & $\begin{array}{l}\text { *Response time difference of one terminals at the same time } \\
* \text { Response time difference of different terminals at the same time }\end{array}$ \\
\hline
\end{tabular}

Fig. 4. Capabilities and items for SOMROMG, MOSROMG, and MOMROMG evaluation, and functional/quantitative evaluation items for intermediate service node of MOMR operation mode

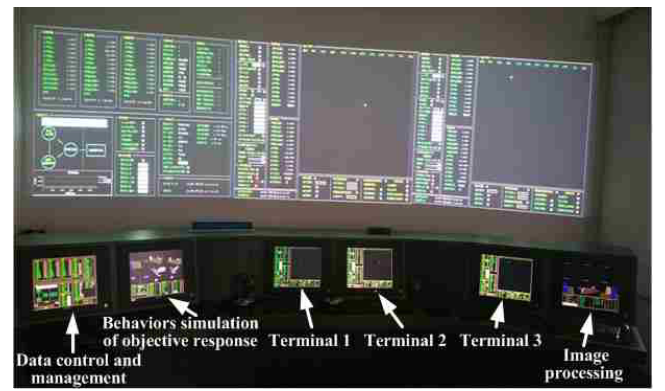

Fig. 5. Some MOSR teleoperation systems

\section{A. Communication capability}

1) Internal/external communication bandwith $(C B)$ :

- *CB among internal units of terminal: $1000 \mathrm{Mbps}$

- ${ }^{*} \mathrm{CB}$ of terminal and object: master channel: $54 \mathrm{Mbps}$ (wireless), slave channel: $100 \mathrm{Mbps}$ (wired)

2) Internal/external communication bit error rate (CBER):

- *CBER among internal units of terminal: $\leq 0.01 \%$

- *CBER of terminal and object: $\leq 0.02 \%$

3) Internal/external communication bit speed (CBS):

- *CBS among internal units of terminal: 27.5 Mbps

- *CBS of terminal and object: $9.3 \mathrm{Mbps}$
4) Internal/external communication packet loss rate $(C P L R)$ :

- CBS among internal units of terminal: $\leq 0.03 \%$

- CBS of terminal and object: $\leq 0.05 \%$

B. Coverage capability of operation mode to teleoperation task

1) Cooperative teleoperation capability (CTA):

- SOMR operation mode: $\square$

- MOSR operation mode: $\square$

- MOMR operation mode: $\otimes$

- Simultaneous operation mode: $\otimes$

- Time sharing operation mode: $\nabla$

2) Operation mode coverage (OMC):

- Autonomous mode(AM): $\square$

- Monitoring mode (MAM): $\square$

- Macro mode (MM): $\square$

- Preprogramming mode (PPM): $\square$

- Numerical interaction mode (NIM): $\square$

- Operator interaction mode (OIM): $\square$

3) Operation mode start (OMS), Operation mode terminal (OMT) and Operation mode switch (OMSW):

- Start at any mode start: $\square$

- Stop at any mode: $\square$

- Switch at any mode : $\square$

- *Elapsed time: shown in Table I. 
TABLE I

ELAPSED TIME OF OPERATION MODE START, STOP, AND SWITCH

\begin{tabular}{|c|c|c|c|c|}
\hline & AM/MM & MAM & PPM & NIM/OIM \\
\hline Start & $4.5 \mathrm{~s}$ & $6.5 \mathrm{~s}$ & $6.5 \mathrm{~s}$ & $7.0 \mathrm{~s} / 9.0 \mathrm{~s}$ \\
\hline Stop & $3.0 \mathrm{~s}$ & $3.0 \mathrm{~s}$ & $3.0 \mathrm{~s}$ & $3.0 \mathrm{~s}$ \\
\hline Switch to AM/MM & $/$ & $4.5 \mathrm{~s}$ & $4.5 \mathrm{~s}$ & $5.5 \mathrm{~s}$ \\
MAM & $5.5 \mathrm{~s}$ & $/$ & $3.5 \mathrm{~s}$ & $5.5 \mathrm{~s}$ \\
PPM & $5.5 \mathrm{~s}$ & $3.5 \mathrm{~s}$ & $/$ & $5.5 \mathrm{~s}$ \\
NIM/OIM & $7.0 \mathrm{~s} / 9.0 \mathrm{~s}$ & $7.0 \mathrm{~s} / 9.0 \mathrm{~s}$ & $7.0 \mathrm{~s} / 9.0 \mathrm{~s}$ & $4.0 \mathrm{~s} / 4.0 \mathrm{~s}$ \\
\hline
\end{tabular}

\section{Systems evaluation}

From the evaluation method described in Section II, the weight matrix and corresponding evaluation value of the first and second universal capability can be obtained as follows.

$$
\begin{gathered}
\mathbf{P}_{1}=\mathbf{0} ; \mathbf{Q}_{1}=\left[\begin{array}{c}
1 \\
\vdots \\
1
\end{array}\right]_{12 \times 1} \\
\mathbf{P}_{2}=[1,1,0,0,1,1,1,1,1,1,1,1]^{\mathrm{T}} ; \mathbf{Q}_{2}=\left[\begin{array}{c}
1 \\
\vdots \\
1
\end{array}\right]_{26 \times 1}
\end{gathered}
$$

Then we can give the corresponding weight matrix of functional and quantitative evaluation items respectively by systems performance requirements. Similarly, the evaluation value and corresponding weight matrix of the rest universal capabilities in Fig. 3, and group evaluation value in Fig. 4 and and corresponding weight matrix for this MOSR teleoperation systems can also be given. After obtaining all evaluation value, the comprehensive systems evaluation can be completed with Eqs. (1) and (2).

\section{CONCLUSION}

On the basis of at least one teleoperation task data, a complete comprehensive evaluation method for MOMR teleoperation systems were given in this paper; besides, the grouping method and corresponding evaluation method of each group are discussed in details. 158 functional evaluation items and 84 quantitative evaluation items covering all capabilities were further summarized for operatio mode group and comprehensive evaluation of MOMR system. This method can be applied to qualitatively understand the functionality completeness and analyze the application effectiveness of a MOMR teleoperation systems, which has a guiding role in the overall design and development of cooperative teleoperation for complex system and multi-object.

\section{ACKNOWLEDGMENT}

Supported by the Strategic Priority Research Program of Chinese Academy of Sciences (Grant No. XDA17030200), National Natural Science Foundation of China (Grant No. 11702294, 11002143), and Key Project of Chinese National Programs for Fundamental Research and Development, 973 Program (Grant No. 2013CB733000).

\section{REFERENCES}

[1] M. Panzirsch, R. Balachandran, and J. Artigas, "Cartesian task allocation for cooperative, multilateral teleoperation under time delay," in IEEE International Conference on Robotics and Automation, 2015, pp. 312317

[2] Y. Zhang, G. Song, H. Sun, Z. Wei, and A. Song, "Design and research on feedback force in bilateral teleoperation system for multiple mobile robots," Dongnan Daxue Xuebao, vol. 47, no. 1, pp. 50-55, 2017.

[3] W. Li, H. Zhang, H. Ma, and X. Xiao, "Space robot reliable teleoperation strategy under large time delay," Journal of Mechanical Engineering, vol. 53, no. 11, pp. 90-96, 2017.

[4] J. J. Roldn, E. Peatapia, A. Martnbarrio, M. A. Olivaresmndez, C. J. Del, and A. Barrientos, "Multi-robot interfaces and operator situational awareness: Study of the impact of immersion and prediction." Sensors, vol. 17 , no. 8, p. $1720,2017$.

[5] P. Setoodeh, S. Sirouspour, and A. Shahdi, "Discrete-time multi-model control for cooperative teleoperation under time delay," in IEEE International Conference on Robotics and Automation, 2006, pp. 2921-2926.

[6] C. Aldana, E. Nuo, and L. Basaez, "Bilateral teleoperation of cooperative manipulators," in IEEE International Conference on Robotics and Automation, 2012, pp. 4274-4279.

[7] D. H. Zhai and Y. Xia, "Adaptive fuzzy control of multilateral asymmetric teleoperation for coordinated multiple mobile manipulators," IEEE Transactions on Fuzzy Systems, vol. 24, no. 1, pp. 57-70, 2016.

[8] C. Yang, X. Wang, Z. Li, Y. Li, and C. Y. Su, "Teleoperation control based on combination of wave variable and neural networks," IEEE Transactions on Systems Man \& Cybernetics Systems, vol. PP, no. 99, pp. 1-12, 2016.

[9] T. Miyoshi, T. Imamura, and K. Terashima, "Design method of multilateral tele-control for multi-client and multi-coupled physical model server," in Advanced Robotics and ITS Social Impacts, 2013, pp. 206-211. 